\title{
Dielectric mismatch effect on coupled shallow impurity states in a semiconductor nanowire
}

\author{
Bin Li, ${ }^{1, *}$ B. Partoens, ${ }^{1}$ F. M. Peeters, ${ }^{1, \dagger}$ and W. Magnus ${ }^{1,2}$ \\ ${ }^{1}$ Departement Fysica, Universiteit Antwerpen, Groenenborgerlaan 171, B-2020 Antwerpen, Belgium \\ ${ }^{2}$ Interuniversity Microelectronics Centre, Kapeldreef 75, B-3001 Leuven, Belgium \\ (Received 25 April 2008; revised manuscript received 20 October 2008; published 9 February 2009)
}

\begin{abstract}
Coupled shallow impurity states in a freestanding semiconductor nanowire and in a semiconductor nanowire surrounded by a metallic gate are studied within the effective-mass approximation. Bonding and antibonding states are found due to the coupling of the two impurities, and their energy converges with increasing distance $d_{i}$ between the two impurities. The dependences of the binding energy on the wire radius $R$, the distance $d_{i}$ between the two impurities, and the impurity radial position in the nanowire are examined.
\end{abstract}

DOI: 10.1103/PhysRevB.79.085306

PACS number(s): 73.22.-f, 73.21.-b, 77.22.-d, 61.46.Km

\section{INTRODUCTION}

Dopant atoms are essential in semiconductor technology since they provide extrinsic charges necessary to create devices such as diodes and transistors. Especially in nanostructures, due to quantum confinement, the position of the dopants can strongly influence the electrical properties of the devices. ${ }^{1}$ Doped nanowires (NWs) in which a dopant is used as the functional part of the device have received considerable attention in recent years due to their potential as building blocks for field-effect transistors (FETs), ${ }^{2,3}$ inverters, ${ }^{4}$ logic circuits, ${ }^{5}$ sensors, ${ }^{6,7}$ and interconnects in nanoelectronics. ${ }^{8}$ The ability to control the location of dopant atoms offers an exciting perspective toward the fabrication of these atomic-scale electronic devices. Atomic control over dopant location in $\mathrm{Si}$ was recently reached by using scanning tunneling microscope lithography, ${ }^{9,10}$ and even precise control of the number and position of the dopants was reported by using single-ion implantation technique. ${ }^{11}$

Due to the dielectric and strain mismatch at the interface of the NWs, the electrical properties of the nanowires will be modified. ${ }^{12}$ The effect of the dielectric confinement on the electronic structure ${ }^{13-15}$ of the NW and on the scattering properties $^{16,17}$ and excitonic absorption spectrum ${ }^{18-20}$ of free electrons in NWs has already been investigated.

Small numbers of impurities, which behave like hydrogen atoms, have the potential to be a testing ground for fundamental questions of nanoscale physics. Coupled impurities have been considered as a functional part in a quantum computer in which the logical information is encoded on electron $\operatorname{spin}^{21}$ or on the charge degrees of freedom. ${ }^{22}$ For example, in the charge-based quantum computing, the lowest two states of a single electron, which are localized by the double well formed by two impurity ions, give rise to a natural identification of the quantum logic states. Due to the importance of coupled impurities in nanotechnology, we will extend our previous study on single shallow impurity states ${ }^{1}$ to two coupled shallow impurities located inside the semiconductor nanowire. We study how the double well which originates from the coupled impurities influences the electron states and how the electron bound states depend on the radial position of the impurities and on the distance between the two impurities. To limit the number of parameters we restrict ourselves to impurities that are positioned on a connecting line which is parallel to the wire axis: the two impurities have the same distance from the interface which is preferable to realize a quantum qubit based on impurity states in semiconductors and is just as in Ref. 22. We consider both a freestanding NW and a NW surrounded by a metallic gate. We use the finite element method (FEM), and the study is done within the effective-mass approximation. ${ }^{23}$ The dependence of the binding energy and the wave function on the wire radius $R$ and on the impurities radial position $\rho_{i}$ will be investigated.

This paper is organized as follows. We present the physical model in Sec. II and give details on the differential equation that we have to solve numerically. The results for the binding energy of freestanding nanowires and nanowires surrounded by a metallic gate are presented in Sec. III. Our conclusions are given in Sec. IV.

\section{MODEL}

A schematic illustration of the model system is shown in Fig. 1. We treat the problem within the effective-mass approximation where the electron has an effective mass $m_{e}^{*}$ and dielectric permittivity $\varepsilon_{1}$ while the surrounding barrier material has a dielectric permittivity $\varepsilon_{2}$. The potential at an arbitrary point inside such a system is the sum of the potential generated by the electron and the two ionized impurities (the two impurities are placed in the $x-z$ plane). The potential

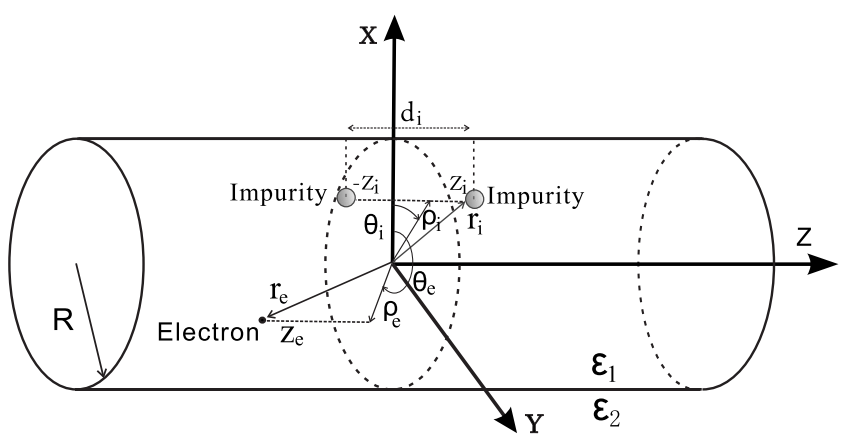

FIG. 1. Schematics of the system: two shallow impurities placed at a distance $d_{i}$ from each other and located at the same radial distance $\rho_{i}$ and parallel to the wire axis. The single electron is assumed to be bound. The nanowire is surrounded by a different dielectric medium. 
generated by a charged particle positioned in $\vec{r}_{q}$ can be obtained by solving the following Poisson equation: ${ }^{16,18}$

$$
\nabla\left[\varepsilon(\rho) \nabla V\left(\vec{r}, \vec{r}_{q}\right)\right]=-q \delta\left(\vec{r}-\vec{r}_{q}\right),
$$

with $\varepsilon(\rho)=\varepsilon_{2}+\left(\varepsilon_{1}-\varepsilon_{2}\right) \theta(R-\rho)$. The potential $V\left(\vec{r}, \vec{r}_{q}\right)$ for a single charged particle was already derived in our previous work. $^{1,13}$ Then the electron potential energy can be obtained by taking the following integral:

$$
U_{\mathrm{tot}}\left(\vec{r}_{e}\right)=-e \int d \vec{r} \delta\left(\vec{r}-\vec{r}_{e}\right)\left[V\left(\vec{r}, \vec{r}_{e}\right)+V\left(\vec{r}, \vec{r}_{q_{1}}, \vec{r}_{q_{2}}\right)\right] .
$$

The first term in the integral is the electrostatic energy due to the image of the electron. As we did before, the electrostatic self-energy coming from part of the first term, which is proportional to $\delta\left(\vec{r}-\vec{r}_{e}\right) /\left|\vec{r}-\vec{r}_{e}\right|$, has been subtracted. The second term is the electrostatic energy due to the two impurities positioned at $\vec{r}_{q_{1}}, \vec{r}_{q_{2}}$, and their images, here $V\left(\vec{r}, \vec{r}_{q_{1}}, \vec{r}_{q_{2}}\right)$ $=V\left(\vec{r}, \vec{r}_{q_{1}}\right)+V\left(\vec{r}, \vec{r}_{q_{2}}\right)$. Then the electrostatic energy of the electron can be rewritten as ${ }^{13}$

$$
U_{\text {tot }}=U_{\text {int }}\left(\vec{r}_{e}, \vec{r}_{q_{1}}, \vec{r}_{q_{2}}\right)+U_{\text {self }}\left(\rho_{e}\right),
$$

which consists of two parts: $U_{\text {int }}\left(\vec{r}_{e}, \vec{r}_{q_{1}}, \vec{r}_{q_{2}}\right)=e\left[V_{\mathrm{im}}\left(\vec{r}_{e}, \vec{r}_{q_{1}}\right)\right.$ $\left.+V_{\text {im }}\left(\vec{r}_{e}, \vec{r}_{q_{2}}\right)\right]$ is the Coulomb potential energy due to the two impurities (which also includes a potential energy that comes from the images of the impurities) and

$$
U_{\text {self }}\left(\rho_{e}\right)=\frac{-e^{2}}{4 \pi^{2} \varepsilon_{1}} \sum_{m=-\infty}^{+\infty} \int_{0}^{+\infty} d k f_{m}\left(\frac{\varepsilon_{1}}{\varepsilon_{2}}, k R\right) I_{m}^{2}\left(k \rho_{e}\right)
$$

is the self-potential energy ${ }^{13}$ with

$$
f_{m}\left(\frac{\varepsilon_{1}}{\varepsilon_{2}}, k R\right)=\frac{\left(1-\frac{\varepsilon_{1}}{\varepsilon_{2}}\right) K_{m}(k R) K_{m}^{\prime}(k R)}{I_{m}(k R) K_{m}^{\prime}(k R)-\frac{\varepsilon_{1}}{\varepsilon_{2}} I_{m}^{\prime}(k R) K_{m}(k R)} .
$$

Moreover the self-energy $U_{\text {self }}\left(\rho_{e}\right)$ is independent of the position of the impurities and depends only on the electron radial coordinate.

Figure 2 shows the total potential energy of the electron in the $x-z$ plane. We can see that when $d_{i}=2 R$, the coupling between the two impurities is weak and they behave like two single impurities. But when $d_{i}$ is small, e.g., $d_{i}=0.2 R$, the coupling between the two impurities will be very large and the total potential energy is very similar to the potential energy of an electron around a single impurity with a double impurity charge. The bottom four figures show contour plots of the potential in the $x-z$ plane, which show the extent of the total potential well. We found that the potential has a larger extent for freestanding nanowires. Moreover, the potential close to the boundary for gated NWs is negative (i.e., attractive), while for freestanding nanowires, it is positive.

Finally, we obtain the Schrödinger equation for the electron inside the nanowire within the effective-mass approximation,
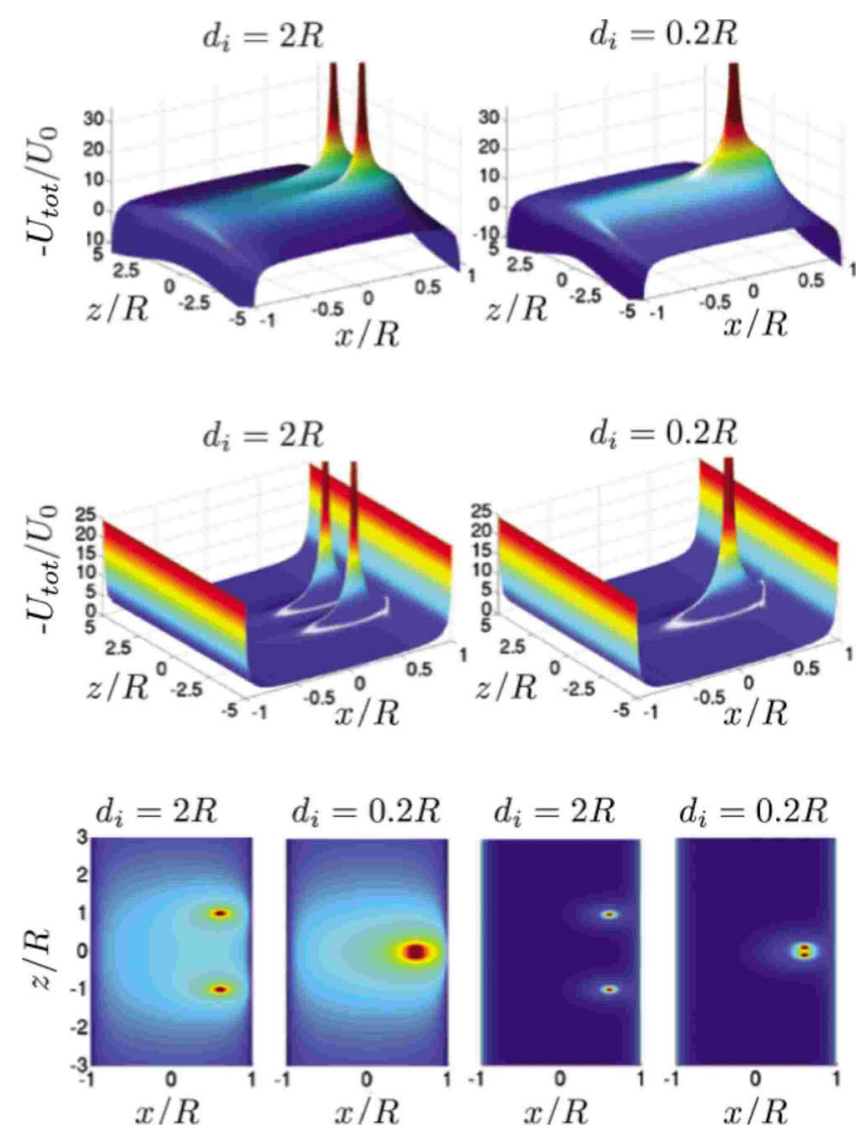

FIG. 2. (Color online) The total potential energy of the electron in the $x-z$ plane. The top two figures are for freestanding nanowires with $d_{i}=2 R$ and $d_{i}=0.2 R$, while the middle two are for gated nanowires. The bottom four figures are contour plots of the electron total potential energy in the $x-z$ plane (the left two are for freestanding nanowires, and the right two are for gated nanowires). Here $U_{0}$ $=e^{2} /\left(4 \pi \varepsilon_{1} R\right)$ is the energy unit.

$$
\left(-\frac{\hbar^{2}}{2 m_{e}^{*}} \nabla_{e}^{2}+U_{\text {int }}\left(\vec{r}_{e}, \vec{r}_{q_{1}}, \vec{r}_{q_{2}}\right)+U_{\text {self }}\left(\rho_{e}\right)+U_{c}\left(\rho_{e}\right)\right) \psi_{e}=E_{\text {tot }} \psi_{e} .
$$

Here $U_{c}\left(\rho_{e}\right)$ is the confinement potential, which is taken to be a circular potential well with infinite height [the assumption will be good enough for freestanding NWs and for gated NWs (Ref. 14)],

$$
U_{c}\left(\rho_{e}\right)= \begin{cases}0, & \rho_{e} \leq R \\ \infty, & \rho_{e}>R\end{cases}
$$

By using the technique of FEM, we are able to solve the resulting three-dimensional (3D) Schrödinger equation in a numerically exact way. The self-energy $U_{\text {self }}\left(\rho_{e}\right)$ will be first calculated and fitted to a function of the electron radial coordinate. The potential energy $U_{\text {int }}\left(\vec{r}_{e}, \vec{r}_{q_{1}}, \vec{r}_{q_{2}}\right)$ will be obtained using a Poisson solver which will give the electrostatic energy between the electron and the two impurities (together with their images). 

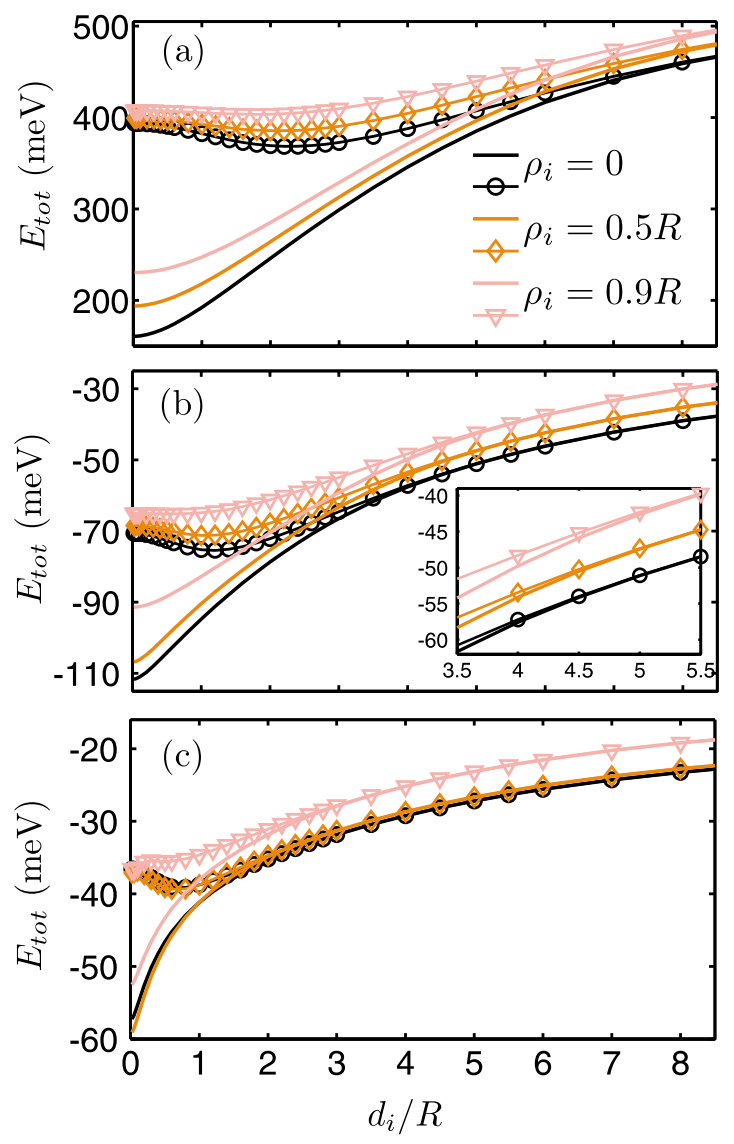

FIG. 3. (Color online) Ground-state energy (solid line) and firstexcited-state energy (solid line with symbol) of the electron in a freestanding GaAs nanowire as a function of the distance between the two impurities and three different radial positions. The radius of the wire (a) $R=2 \mathrm{~nm}$, (b) $R=10 \mathrm{~nm}$, and (c) $R=30 \mathrm{~nm}$. Inset: enlargement of (b) around $d_{i}=4.5 R$.

\section{RESULTS AND DISCUSSIONS}

\section{A. Freestanding nanowires}

As an example, we consider first a freestanding GaAs nanowire which has a dielectric permittivity $\varepsilon_{1}=12.5 \varepsilon_{0}$ and an effective electron mass $m_{e}^{*}=0.067 m_{e}$ and $\varepsilon_{2}=\varepsilon_{0}=1$. The ground-state and first-excited-state energies are shown in Fig. 3. Figures 3(a)-3(c) correspond to NWs with radius $R$ $=2,10$, and $30 \mathrm{~nm}$, respectively. Note that we did not include the impurity-impurity interaction to these total energies as it gives no contribution to the binding energy. The ground (first excited) state corresponds to the bonding (antibonding) state which is symmetric (antisymmetric) with respect to the plane $z=0$. For small distance between the impurities there is a large energy gap between the bonding and antibonding states, which closes with increasing $d_{i}$. For large $d_{i}$ the bonding and antibonding states have the same energy, which is equal to the energy of an electron localized on top of one of the impurities. Compared to the results of a single impurity ${ }^{1}$ we found that the total energies are much smaller (e.g., for very small $d_{i}$, the total energy is less than one-fourth of the one for a single impurity). From the three figures we conclude that the smaller the radius, the faster the bonding and
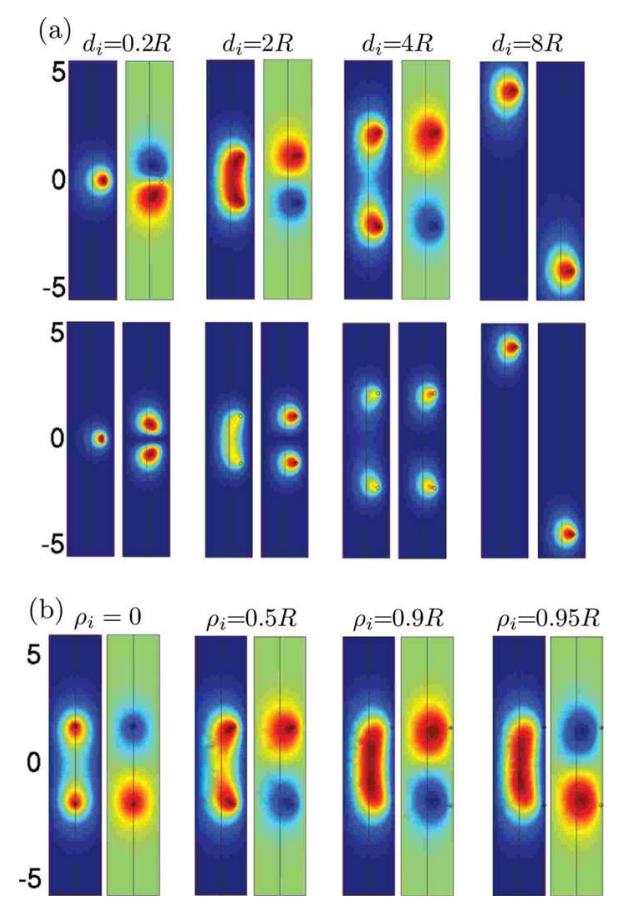

FIG. 4. (Color online) (a) Top figures are contour plots of the ground-state wave function (left figure) and the first-excited-state wave function (right figure) of an electron in a freestanding GaAs nanowire with radius $R=10 \mathrm{~nm}$ and containing two impurities fixed at $0.5 R$ from the center. The top four couples of figures from left to right are for interimpurity distance: $d_{i}=0.2 R, 2 R, 4 R$, and $8 R$. The bottom figures are the corresponding probability of the electron in the $x-z$ plane. (b) The same as (a) but now for fixed $R=10 \mathrm{~nm}$ and $d_{i}=3 R$ and four different radial impurity positions $\rho_{i}=0,0.5 R$, $0.9 R$, and $0.95 R$.

antibonding states overlap [we can see from Fig. 3(a) that the bonding state and antibonding state of $\rho_{i}=0$ start to overlap when $d_{i} \approx 8 R=16 \mathrm{~nm}$, while from Fig. 3(b) we find that the same behavior starts at $\left.d_{i} \approx 4 R=40 \mathrm{~nm}\right]$. Thus when the radius of the wire is smaller, due to the larger lateral localization, the electron tends to localize at the position of the impurities and form a degenerate bonding-antibonding ground state. Furthermore, the inset of Fig. 3(b) shows that the bonding and antibonding state energies will overlap slower when the impurity is closer to the interface.

A contour plot in the $x-z$ plane of the ground-state (bonding state) wave function and the first-excited-state (antibonding state) wave function is shown for four different interimpurity distances $d_{i}$ when $R=10 \mathrm{~nm}$ and $\rho_{i}=0.5 R$ in Fig. 4(a) and in Fig. 4(b) four different impurity positions when $R$ $=30 \mathrm{~nm}$ and $d_{i}=2 R$. Here we should mention that since the color that indicates zero is different in each figure, the colors in these figures rather accentuate the localization of the electron. The top figures in Fig. 4(a) show clearly that when $d_{i}$ $=0.2 R$ the shapes of the bonding and the antibonding states (also the probability of the electron shown in the bottom figures) are very similar to the two lowest states of a single impurity, which means that the two impurities are strongly coupled and they behave like a single impurity with two unit charges. When $d_{i}=2 R$ the shape of the ground state becomes more extended in the $z$ direction and has two centers, but the 

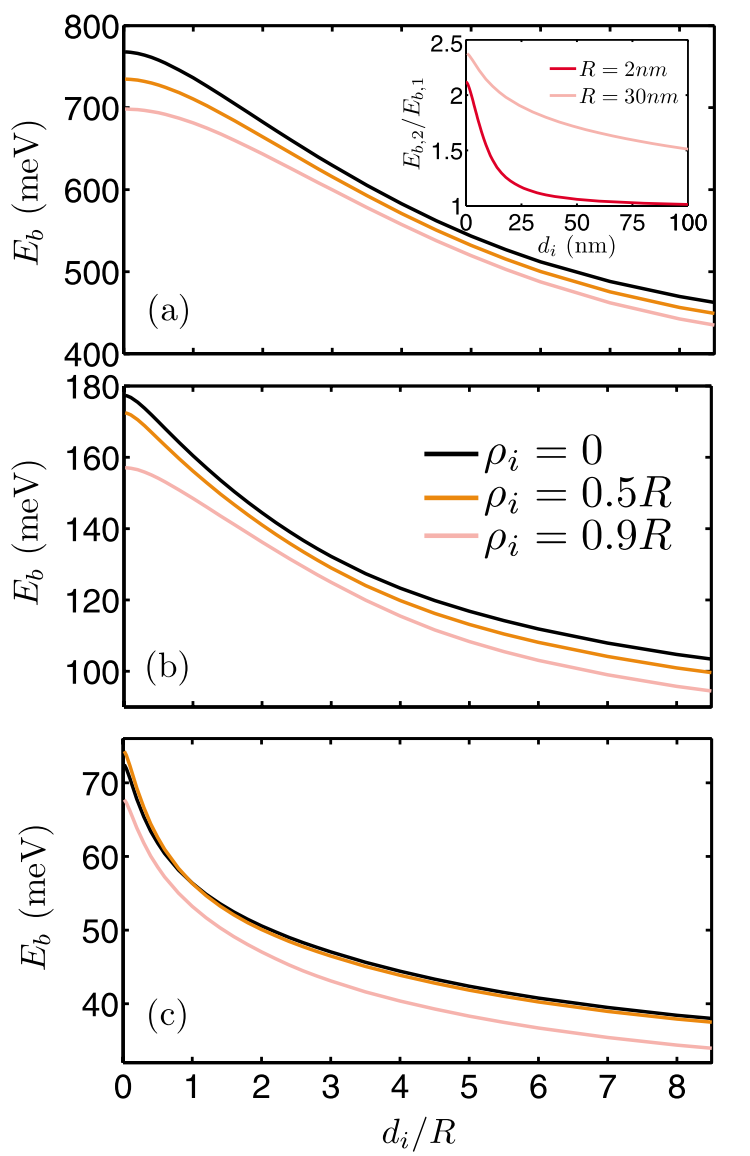

FIG. 5. (Color online) The binding energies of a freestanding GaAs nanowire with two impurities fixed at the center and $0.5 R$ and $0.9 R$ from the center of the wire. (a), (b), and (c) are for $R=2,10$, and $30 \mathrm{~nm}$, respectively. Inset: the binding energy of a freestanding GaAs nanowire in the case of two coupled impurities divided by the one with a single impurity for $R=2 \mathrm{~nm}$ and $R=30 \mathrm{~nm}$, with $\rho_{i}$ $=0$.

two impurities are still strongly coupled. Then for $d_{i}=4 R$ the coupling becomes weak and the shapes of the bonding state and the antibonding state become similar, which is seen more clearly from the contour plot of the probability of the electron [bottom contour plots in Fig. 4(a)]. Finally when the distance between the two impurities is very large, e.g., $d_{i}$ $=8 R$, the bonding and the antibonding states become identical within our numerical accuracy. Here for $d_{i}=8 R$, we found numerically that for the lowest two energy levels the electron is localized on one of the impurities. Such a state is not the ground state, but the energy difference with the symmetric bonding state is extremely small. Figure 4(b) shows the wave functions for different radial positions of the impurities. We can see that the bonding and antibonding states differ less for smaller values of $\rho_{i}$. When the impurities are closer to the boundary of the wire the bonding and antibonding states are more differentiated, which is a result of the strong confinement: the confinement will push the electron away from the interface, thus away from the impurities. Then the localization of the electron on either of the impurities will be weaker. The same effect is found for gated NWs (Sec. IV).

Next, let us look at the behavior of the binding energy, which is the total energy of the two impurities and the energy
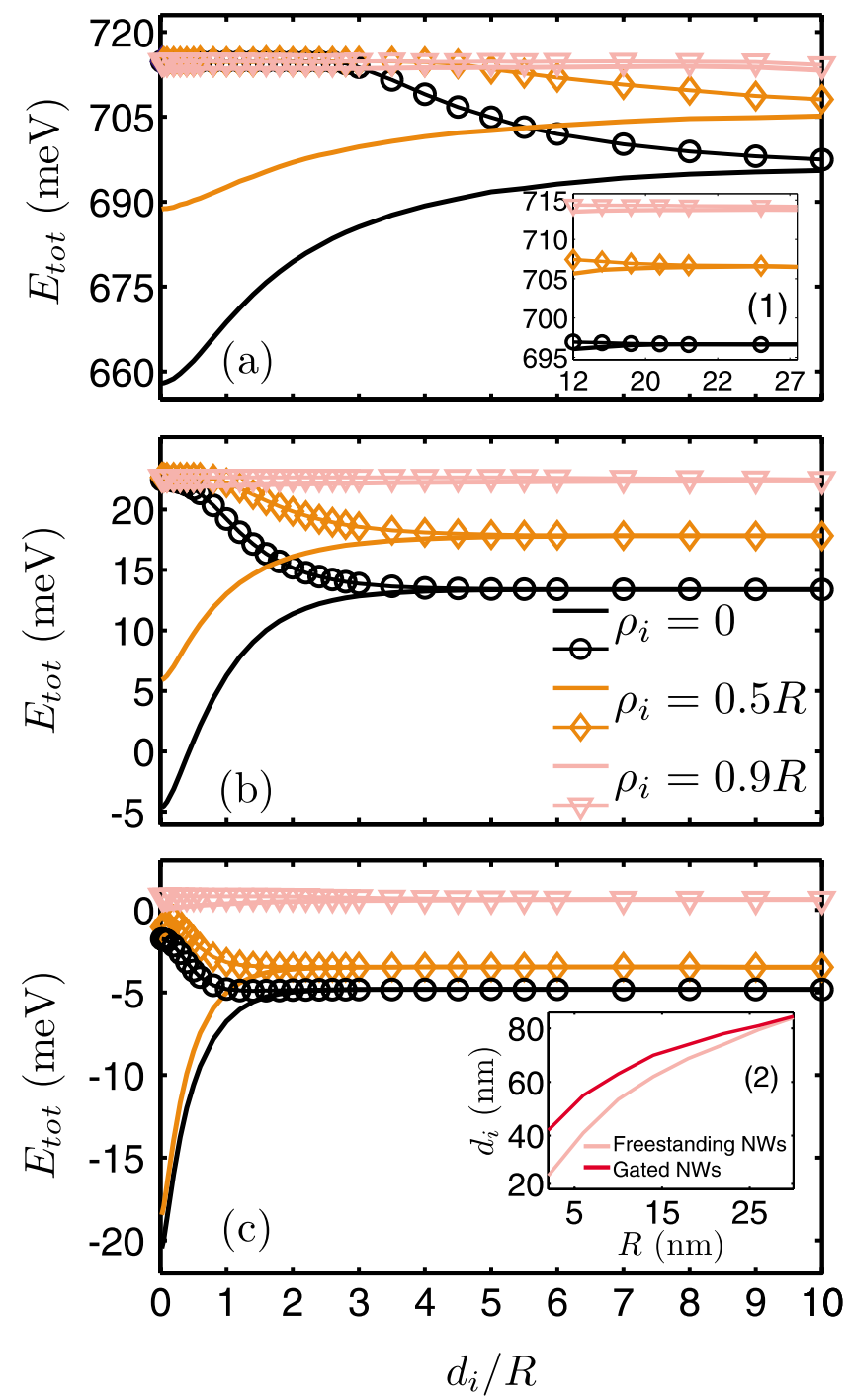

FIG. 6. (Color online) (a) Ground-state (solid line) and firstexcited-state (solid line with symbol) energies of an electron in a GaAs nanowire with radius $R=2 \mathrm{~nm}$ surrounded by a metallic gate and coupled to two impurities. (b) The same as (a) but now for $R$ $=10 \mathrm{~nm}$ and (c) for $R=30 \mathrm{~nm}$. Inset (1): same as (a) but for a larger range of $d_{i}$; inset (2) $d_{i}$ at which the bonding and antibonding energies are the same, within $0.5 \%$, as a function of the radius $R$, here $\rho_{i}=0$.

of an unbound electron minus the total energy of the bound system (total energy of the electron, the two impurities, and their images). ${ }^{1}$ Figures 5(a)-5(c) give the binding energies for nanowires with radius $R=2,10$, and $30 \mathrm{~nm}$, respectively. Notice that the larger the distance $d_{i}$ between the two impurities, the smaller the binding energy becomes. From the inset of Fig. 5(a) we see that the binding energy for freestanding nanowires with two impurities is much larger than for the ones with a single impurity, but due to the dielectric mismatch effect this ratio is much smaller than 4 (which is the value of the binding energy of an impurity with two unit charges divided by the one with a single unit charge in the absence of any dielectric mismatch effect). For example, when $R=2 \mathrm{~nm}$ and $\rho_{i}=0$ the binding energy could be nearly $800 \mathrm{meV}$ which is about 2.5 times the value of the one with 


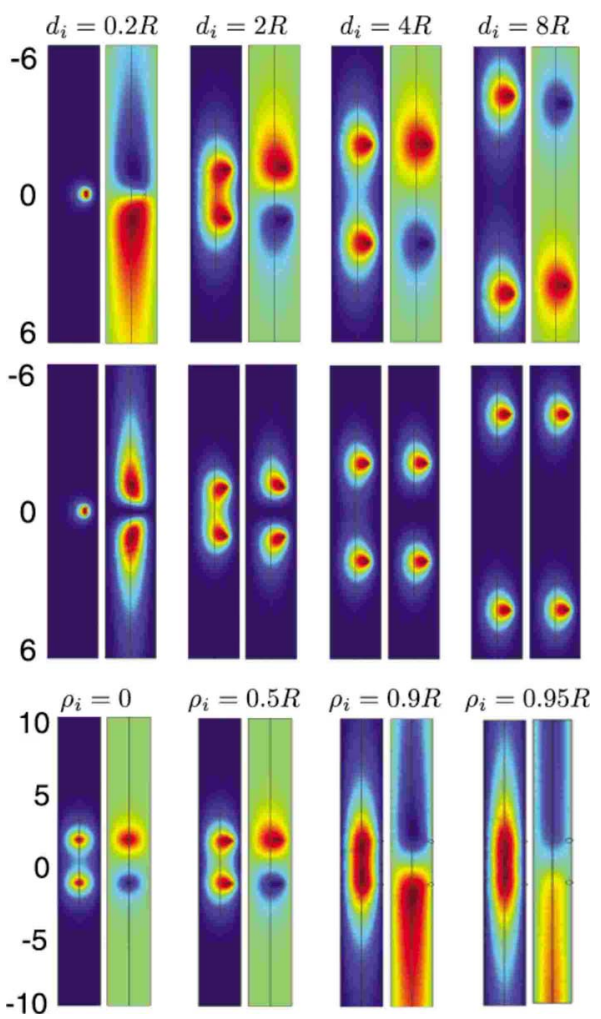

FIG. 7. (Color online) (a) Top figures are contour plots of the ground-state and the first-excited-state wave functions of an electron in a gated GaAs nanowire with radius $R=10 \mathrm{~nm}$ and containing two impurities fixed at $0.5 R$ from the center of the wire. The top four figures from left to right are for interimpurity distance: $d_{i}$ $=0.2,2 R, 4 R$, and $8 R$, respectively. The bottom figures are the corresponding probability of the electron in the $x-z$ plane. (b) The same as (a) but now for fixed $R=10 \mathrm{~nm}$ and $d_{i}=3 R$ and four different radial impurity positions $\rho_{i}=0,0.5 R, 0.9 R$, and $0.95 R$.

a single impurity. For small $R$ the screening due to the dielectric mismatch is much more significantly reducing the ratio $E_{b, 2} / E_{b, 1}$ by increasing the distance between the two impurities and also leading to a faster decrease in the interimpurity coupling. We notice from Fig. 5(c) an interesting effect for $d_{i}<R$ : the binding energy for $\rho_{i}=0.5 R$ is larger than for $\rho_{i}=0$, which was not found in Ref. 1 for the case of a nanowire with a single impurity. The reason is that for larger $R$, the repulsive Coulomb interaction from the image charge will push the electron away from the interface, but as the Coulomb interaction between the two impurities is large (especially for smaller $d_{i}$ ), this repulsion from the interface enhances the localization of the electron on the impurity site for larger $\rho_{i}$, leading to a larger binding energy.

\section{B. Gated nanowires}

For gated GaAs nanowires, the dielectric permittivity $\varepsilon_{2}$ of the surrounding barrier material will tend to infinity. The total energy is shown in Fig. 6. The difference from freestanding NWs (see Fig. 3) is that the ground-state and the first-excited-state energies tend to overlap more slowly with increasing $d_{i}$, which means that the two impurities act as a whole for a larger range of $d_{i}$ in case a metallic gate is present. This is a consequence of the fact that for gated NWs the image charges of the two impurities are negative, which tends to push the electron toward the region between the two impurities. However, for gated NWs, when the bonding and antibonding state energies become (almost) equal, they have reached a constant value, which is actually the energy of NWs with a single impurity. This is much faster than for the freestanding NWs. Thus the coupling between the two impurities decreases faster for gated nanowires and appears at a smaller range of $d_{i}$. The reason is that when the distance $d_{i}$ between the two impurities is very large and the electron is close to one of the impurity sites, the total Coulomb energy from the other impurity and the images of the other impurity will be almost zero; thus the contribution from the other impurity to the total energy is negligible. Whereas for freestanding nanowires, since the Coulomb energy from the image of the impurity is also negative, there will still exist a

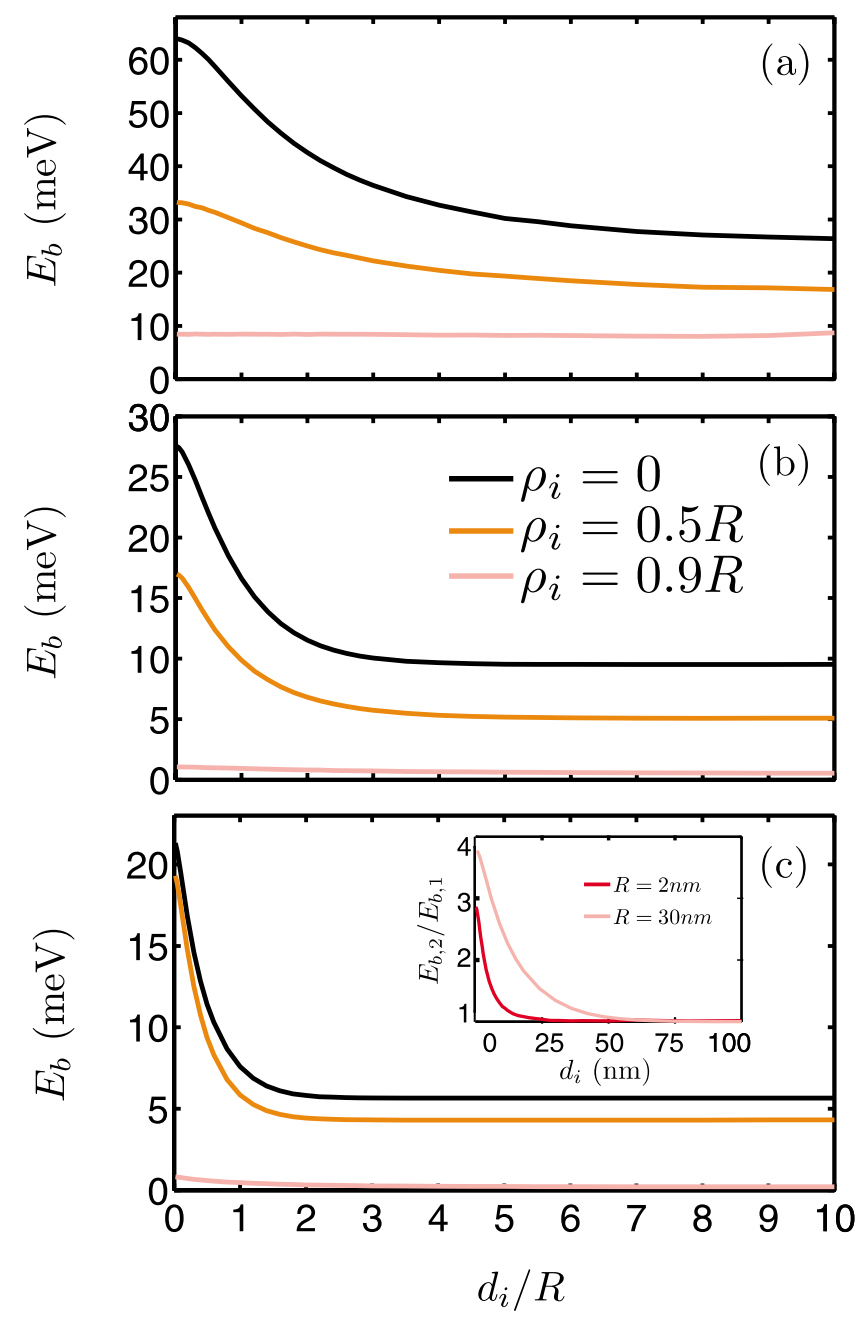

FIG. 8. (Color online) (a) The binding energies of an electron in a GaAs nanowire surrounded by a metallic gate with two impurities fixed at the center and $0.5 R$ from the center. Here the radius of the wire is $R=2 \mathrm{~nm}$; [(b) and (c)] the same as (a), but here the radii is $R=10 \mathrm{~nm}$ and $R=30 \mathrm{~nm}$, respectively. Inset: the binding energy of gated GaAs NW with two impurities relative to that of the one with a single impurity for $R=2 \mathrm{~nm}$ and $R=30 \mathrm{~nm}$, with $\rho_{i}=0$. 
contribution from the other impurity to the total energy when distance $d_{i}$ is large, and this contribution will decrease with increasing $d_{i}$. As a result the curves in Figs. 3(a)-3(c) will tend to a constant value much slower. From inset (2) of Fig. 6 we can clearly see that the distance $d_{i}$ between the two impurities, at which the bonding state and the antibonding state converges, is larger for gated nanowires than for freestanding nanowires, but it increases more slowly.

Figure 7 shows contour plots of the ground-state (bonding state) wave function and the first-excited-state (antibonding state) wave function of an electron in the $x-z$ plane for a gated GaAs nanowire which contains two impurities fixed at $0.5 R$ from the center. As previously for freestanding nanowires, the colors in these figures rather accentuate the localization of the electron. Compared to nongated GaAs nanowires we found similar results except that (1) the bonding and antibonding states are more extended in the $z$ direction, especially for smaller values of $d_{i}$ and larger values of $\rho_{i}$ and (2) due to the screening effect of the metallic gate, the two impurities will be more weakly coupled as compared to the freestanding nanowires (clearly shown in the figures for $d_{i}$ $=2 R$ ). Here we should point out that since gated NWs have a larger energy difference of the bonding and antibonding states, our calculation can still clearly distinguish these two states for large $d_{i}$ (as shown in the figure for $d_{i}=8 R$ ), but for freestanding NWs the energy splitting is too small for our FEM method to tell the difference between the two lowest energy states.

The binding energy for the GaAs nanowires surrounded by a metallic gate is shown in Fig. 8. Figures $8(a), 8(b)$, and 8(c) are for $R=2, R=10 \mathrm{~nm}$, and $R=30 \mathrm{~nm}$, respectively. As compared to the result for a freestanding NW, we found: (1) for GaAs nanowires surrounded by a metallic gate the binding energy decreases fast with increasing distance $d_{i}$ and then quickly tends to a constant value when $d_{i}$ is large; (2) notice from Fig. 8 that the binding energy will be almost zero when $\rho_{i}=0.9 R$, implying a very weak bound state. Whereas in freestanding nanowires there always exists a well-defined bound state. Moreover we can also see that the binding energy in Gated NWs depends more largely on the impurity radial position. Moreover, from the inset of Fig. 8(c) we see that the binding energy for gated nanowires with two impurities can be up to 3.9 times the one for a single impurity when $R$ $=30 \mathrm{~nm}$ (which means the dielectric mismatch effect is almost negligible), which compares to a value of 2.4 for freestanding NWs. The reason is that for gated nanowires, as the images of the impurities are negative, the two impurities tend to localize the electrons much more in both the lateral and $z$ direction as compared to the case of a single impurity. What is more, compared to the inset of Fig. 5(a), we notice that the ratio tends to 1 much faster for gated nanowires; the reason is the same as the one we had given previously to explain the convergence of the two lowest energy states in gated NWs.

\section{CONCLUSIONS}

We studied the electron states inside a semiconductor nanowire, which has two impurities placed at a distance $d_{i}$ and both are at the same radial position $\rho_{i}$. The two impurities lead to bonding and antibonding electron states, and the energy of these two states will converge to each other with increasing distance $d_{i}$ between the two impurities. Moreover, the bonding and antibonding states will converge faster for smaller wire radius $R$ and for smaller values of $\rho_{i}$. We found that the convergence of the bonding and antibonding states will be faster for freestanding nanowires than for gated nanowires with increasing distance $d_{i}$, but the binding energy of these two states will tend to a constant value faster for gated nanowires.

We also investigated the electron binding energy for both freestanding nanowires and gated nanowires, and we found that there is a larger binding-energy difference of different impurity radial positions for gated NWs and its binding energy will tend to a constant value much faster. Moreover, the previously defined ratio $E_{b, 2} / E_{b, 1}$ is much larger for gated NWs than for freestanding NWs.

\section{ACKNOWLEDGMENTS}

This work was supported by the EU-NoE: SANDiE, the Flemish Science Foundation (FWO-V1), the Interuniversity Attraction Poles, Belgium State, Belgium Science Policy, and IMEC, vzW.

\footnotetext{
*phymilky@gmail.com

†rancois.peeters@ua.ac.be

${ }^{1}$ Bin Li, A. F. Slachmuylders, B. Partoens, W. Magnus, and F. M. Peeters, Phys. Rev. B 77, 115335 (2008).

${ }^{2}$ Yat Li, Jie Xiang, Fang Qian, Silvija Gradecak, Yue Wu, Hao Yan, Douglas A. Blom, and Charles M. Lieber, Nano Lett. 7, 6 (2006).

${ }^{3}$ Josh Goldberger, Allon I. Hochbaum, Rong Fan, and Peidong Yang, Nano Lett. 6, 973 (2006).

${ }^{4}$ Y. Cui and C. M. Lieber, Science 291, 851 (2001).

${ }^{5}$ Y. Huang, X. Duan, Y. Cui, L. J. Lauhon, K. H. Kim, and C. M. Lieber, Science 302, 1313 (2003).

${ }^{6}$ H. G. Liu, K. Jun, D. A. Czarplewski, and H. G. Craighead,
}

Nano Lett. 4, 671 (2004).

${ }^{7}$ Y. Cui, Q. Wei, H. Park, and C. M. Lieber, Science 293, 1289 (2001).

${ }^{8}$ G. Zheng, W. Lu, S. Jin, and C. M. Lieber, Adv. Mater. (Weinheim, Ger.) 16, 1890 (2004).

${ }^{9}$ S. R. Schofield, N. J. Curson, M. Y. Simmons, F. J. Ruess, T. Hallam, L. Oberbeck, and R. G. Clark, Phys. Rev. Lett. 91, 136104 (2003).

${ }^{10}$ F. J. Rueß, W. Pok, T. C. G. Reusch, M. J. Butcher, K. E. J. Goh, L. Oberbeck, G. Scappucci, A. R. Hamilton, and M. Y. Simmons, Small 3, 563 (2007).

${ }^{11}$ T. Shinada, S. Okamoto, T. Kobayashi, and L. Ohdomari, Nature (London) 437, 1128 (2005). 
${ }^{12}$ A. R. Smith and H. Ahmed, J. Appl. Phys. 81, 2699 (1997).

${ }^{13}$ A. F. Slachmuylders, B. Partoens, W. Magnus, and F. M. Peeters, Phys. Rev. B 74, 235321 (2006).

${ }^{14}$ A. F. Slachmuylders, B. Partoens, W. Magnus, and F. M. Peeters, Appl. Phys. Lett. 92, 083104 (2008).

${ }^{15}$ M. Diarra, Y. M. Niquet, C. Delerue, and G. Allan, Phys. Rev. B 75, 045301 (2007).

${ }^{16}$ P. Vagner and M. Moško, J. Appl. Phys. 81, 3196 (1997).

${ }^{17}$ J. Planelles and J. L. Movilla, Phys. Rev. B 73, 235350 (2006).

${ }^{18}$ P. Vagner, D. Munzar, and M. Moško, Acta Phys. Pol. A 92, 1038 (1997).
${ }^{19}$ J. L. Movilla and J. Planelles, Phys. Rev. B 75, 195336 (2007).

${ }^{20}$ M. Mosko, D. Munzar, and P. Vagner, Phys. Rev. B 55, 15416 (1997).

${ }^{21}$ M. J. Calderón, Belita Koiller, and S. Das Sarma, Phys. Rev. B 75, 125311 (2007).

${ }^{22}$ L. C. L. Hollenberg, A. S. Dzurak, C. Wellard, A. R. Hamilton, D. J. Reilly, G. J. Milburn, and R. G. Clark, Phys. Rev. B 69, 113301 (2004).

${ }^{23}$ M. Royo, J. Planelles, and M. Pi, Phys. Rev. B 75, 033302 (2007). 